\title{
Assessing the Benefits of a Teleassessment Solution Using a FVM Perspective
}

\author{
Amir Eslami Andargoli \\ Oren Tirosh \\ Nilmini Wickramasinghe \\ Swinburne University of Technology Swinburne University of Technology Swinburne University of Technology \\ aandargoli@swin.edu.au_otirosh@swin.edu.au \\ and Epworth HealthCare \\ nilmini.work@gmail.com
}

\begin{abstract}
The recent COVID-19 pandemic has served to highlight the benefits of digital health in general and telehealth in particular. One area of telehealth that is particularly important is that of teleassessment. Currently, we are witnessing an exponential growth in total knee and total hip replacements (TKR) (THR) due to an aging population coupled with longer life expectancy which is leading to a high likelihood of an unsustainable burden for healthcare delivery in Australia. To address this imminent challenge, the following proffers a tele-assessment solution, ARIADNE (Assist foR hIp AnD kNEe), that can provide high quality care, with access for all and support for high value outcomes. A fit viability assessment is provided to demonstrate benefits of the proffered solution.
\end{abstract}

\section{Introduction}

In Australia, the exponential growth of joint replacements, in particular total hip and total knee replacements (THR) (TKR), is projected to reach an unsustainable burden by 2030 [1], which has many severe and far reaching implications for healthcare delivery and for the demand on public and private hospitals. Given several key contributing factors, most notably an aging population and longer life expectancy [2] , the most prudent way to address this is to leverage technology solutions that can support cost-effective, efficient and effective care delivery post-surgery. We proffer tele-assessment, a noted void in current telemedicine solutions for orthopaedic care, as such a solution.

A central aspect in the recovery from THR and TKR is the return to appropriate postural and functional control [3]. The current standard clinical pathway involves 12 to 60 face-to-face visits over a period of three months [4]. This is not only costly and difficult to manage, especially for isolated and disadvantaged populations [4], but if not done successfully leads to poor clinical outcomes and low patient satisfaction [5]. Moreover, clinical best practice notes that this 5-month window post-surgery is imperative for optimal recovery and best results [6]. To address this critical aspect on the THR and TKR patient journey, and support quality clinical outcomes and patient satisfaction as well as ease the burden for our healthcare system, we design, develop and test ARIADNE (Assist foR hIp AnD kNEe), a pervasive tele-assessment solution that can perform clinical tele-assessment to assess postural and functional control to support post-surgery THR and TKR recovery. ARIADNE will enable objective, remote examination and monitoring of patient functional performance during their typically long rehabilitation journey, something that to date is missing from current telemedicine solutions especially in orthopaedic care (see Figure 1). By implementing such a pervasive tele-assessment solution within traditional practice, we have the potential to: a) improve existing practice patterns, b) shorten the recovery trajectory, c) increase the likelihood for optimal clinical outcomes, and d) support a superior patient experience.

Figure 1. Current standard care and telemedicine

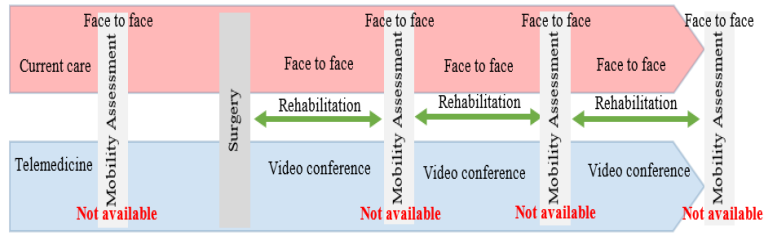

care pathway in orthopaedics.

\subsection{Background}

Total hip or knee replacement is a common surgical intervention for treating advanced hip/knee Osteoarthritis (OA). As a strategy to address the burden of disease of OA in Victoria and optimally align health services to consumers' needs and evidence, the Department of Health and Human Services commissioned the development of a Model of Care $(\mathrm{MoC})$ for Osteoarthritis of the Hip and Knee. A MoC is an evidence and consultationinformed framework that describes what and how health services and other resources should be 
delivered locally to people who live with specific health conditions. In 2018[7], the MoC recommended the Innovation in service delivery model. The model was designed to establish: 1) telehealth services to improve consumers' access to specialist clinics for the purposes of clinical assessment, management planning and treatment, and 2) web-based and smartphone app tools that deliver accurate health information and support behaviour change to consumers and care providers. The development of ARIADNE is designed to addresses the above acknowledgement of the importance of a telemedicine platform to improve health care services for rehabilitation following THR and TKR [7][8].

Tele-rehabilitation via online video communication is an emerging area attracting increased attention as a potential alternative to conventional, face to face rehabilitation, suggested to be an option for people located remotely to reduce the need for frequent travel [9]. A recent systematic review concluded that tele-rehabilitation can lead to better healthcare at lower costs [10]. An example is the tele-rehabilitation eHAB (NeoRehab, Brisbane, Australia) that enables real-time video conferencing to the patient's home and includes features such as recording instruction and exercises [11]. Similarly, MyRehab offers a tele-rehabilitation communication system via text or voice messages and video-conference, currently evaluated in a RCT with THR and TKR patients [12].

Indeed, tele-rehabilitation partially addresses some of the requirements of the MoC. However, a critical missing element in current solutions is teleassessment which supports an objective remote postural and functional assessment integrated with web-based management and planning capabilities. ARIADNE addresses this key void by being able to transform standard care with a face to face assessment, mostly available only in major cities with experts, to provide remote assessment access and quality of care to a wider and remote community. Thus, ARIADNE, will significantly enhance Australian health care services, ensuring objective postural and functional examination can be performed. It will provide the foundation for future telemedicine platforms for clinical trials and treatment monitoring.

To further improve upon the Australian telehealth system the following objectives need to be addressed:

1. An examination into the measurement consistency and agreement of a newly established tele-assessment system with respect to a face to face clinical based reference condition.

2. A determination of the feasibility and the extent to which the tele-assessment can be used by clinicians and patients to achieve effectiveness (accuracy and completeness), efficiency (resources needed for effectiveness) and satisfaction (comfort and acceptability).

3. An assessment of the cost-effectiveness associated with tele-assessment including those related to healthcare, purchase of equipment, mobile phone data usage, and costs associated with establishing and delivering the service, and analysing the results.

\subsection{Theoretical lens}

It is essential for rigor in digital health solution design and development to use a suitable theoretical lens to analyse and assess the fidelity, fit and benefits of the proffered solution [13]. The healthcare industry is challenged by ever-increasing constraints of resources. This puts the viability of digital technological solutions and their implementation at the forefront [14]. At the same time, these technologies should fit within the portfolio of individual and organisational needs [14]. Therefore, for the context of the current study, the Fit-viability model has been chosen to assess the fidelity, fit and viability of the proposed ARIADNE solution.

\subsection{Fit-Viability Model}

Tjan [15] proposed fit viability dimensions for evaluating Internet initiative projects. Liang and Wei [16] by taking these two dimensions and adding Task Technology Fit (TTF) theory proposed a fit-viability model to study m-commerce applications. In their framework, viability measures the readiness of the organization for the technology adoption and implementation, and fit measures capabilities of the systems to optimally perform the required tasks. These two dimensions make a simple matrix with fit on horizontal and viability on vertical axis. By using the four corners of the matrix, organizations can make an informed decision for technology adoption and implementation.

\subsection{Task-Technology Fit}

The theoretical basis of the fit construct is derived from the Task-Technology Fit model which according to Goodhue [17] argues that a fit between task characteristics and system features need to be high for the better performance and success and this will have effect on the decisionmaking process of an organization. Research [18] has indicated that if a system is more aligned with the requirements of the users there are greater chances of system success which leads to better performance. It means that if the features offered 
by the system fit with the task requirements the users will be more incline to use it. Therefore, the success of the system lies in understanding of the affordances from different stakeholders' perspectives [14], and the fit between these affordances [19].

\subsection{Viability}

Viability refers to the degree of impact of environment and organizational factors on a system adoption and implementation decision. Viability of digital technology implementation is bound by a set of constraints which can be adjusted such as technological, organisational and societal constraints [14]. These factors at the macro level include political and social, economic, environmental as well as infrastructure/technology factors. At the organizational level literature has proposed many factors at the strategic and tactical levels [20]. These factors include leadership, management style, polices, information sharing, training and learning, technical staff, and user behaviour. Taking the example of teleassessment, economic and technological factors are crucial factors in digital health implementations; and ignoring these factors could lead to unsuccessful projects. Management support, physical and IT infrastructure create stronger desire of system implementation and innovation adoption, that positively impact viability of the system.

\subsection{Research Framework}

Taking the above components of the theoretical lens, the research framework as shown in figure 2 has been developed to illustrate the key constructs and factors affecting the use of teleassessment for TKR or THR. Fit is measured by matching the requirements of functionality for movement enabled via the teleassessment solution e.g. data format, meeting required operating procedures, and output format. Viability is measured by assessing the impact of these factors on the recovery of the individual user and adoption by physiotherapist.

\subsection{Overview of ARIADNE}

In orthopaedics, performance measures following THR and TKR are required to identify patient functional competency and physical progress. In existing clinical practice, these postural and functional measures include: 1) range of motion, 2) postural balance, 3) chair rise, 4) 40 meter fast paced walk, and 5) timed up and go (TUG) [21], and are executed face to face while the clinician manually records the duration and number of repetitions to complete the task. A more robust objective, but to date only used in research and not in clinical settings due to availability and accessibility, is quantifying performance using Inertial Measuring Units (IMU) motion sensors comprising accelerometers and gyroscopes [22] to measure linear acceleration and angular velocity, respectively. Once the raw data is captured, the level of performance is quantified by a further welldefined signal processing methods [23][24].

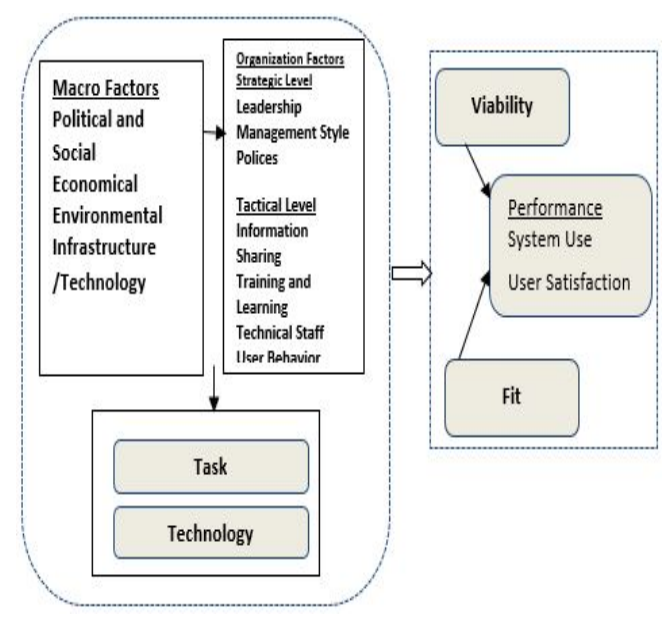

Figure 2: Research Framework

ARIADNE has been developed and designed to support the above requirements and is built from previous work by one of the authors around web based repository applications Gaitabase and PROMsBase [25][26], and leverages his research on the use of IMU motion sensor signals to capture, process, and interpret postural and functional performance [27][28][29]. Gaitabase has been used by world leading gait laboratories for clinical gait analysis, having 22 different centres in 8 different countries on four different continents. PROMsBase is routinely used at Western Health in Victoria to collect patients' satisfaction and wellbeing data pre and post joint replacement procedures with over 8,000 questionnaires from over 10,000 surgery procedures now collected.

\subsection{Development of ARIADNE}

In order to be clinically useful as a teleassessment platform, we extended the technology with unique integration methods of the web based repository system coupled with the motion sensor IMU data captured from a mobile phone. During assessment, the clinician remotely connects to the motion capture app installed on the patient's mobile phone that is strapped at the lower back (to measure postural control) or above the ankle (to measure joint angle) using an ankle strap (figure $3 \mathrm{~A}$ ). Once connected, the clinician remotely operates the app while the patient performs the specific functional 
task as instructed by the clinician (figure 3B). Once the task is completed the clinician remotely saves the mobile sensors data that is automatically uploaded to the web based application for further analysis to generate report on performance compared to healthy population (figure $3 \mathrm{C}$ ). Both the clinician and the patient can login to the web application to evaluate performance and progress.

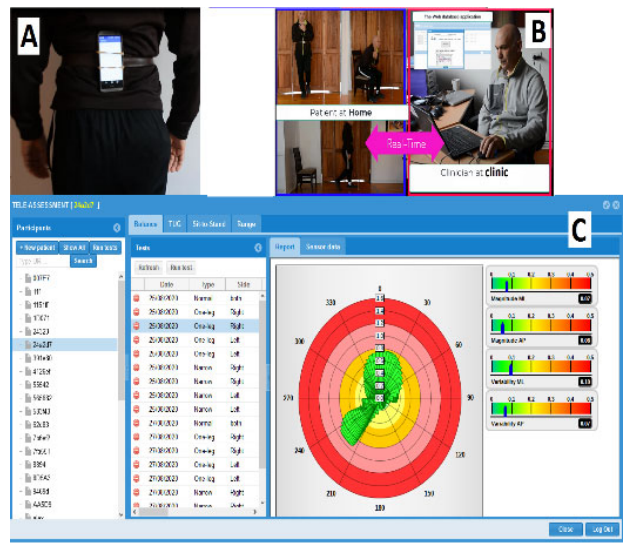

Figure 3. Smartphone placement (A), remote tele-assessment of balance, sit-stand and TUG tests (B), and the web based interface with balance report $(\mathrm{C})$.

\subsection{Validity and Reliability}

The validity and reliability of using IMU to measure the above 5 postural and functional assessments is well documented in a range of population type including: healthy older adults [30], Parkinson disease [31] and TKR [22], showing very good to excellent validity and reliability. These validity and reliability studies and the systematic review [30] used mobile phones motion sensors suggesting that mobile phone are non-inferior when compared to the other postural and functional measurement techniques. Furthermore, our pilot work validating our tele-assessment knee range with the golden standard video analysis also showed excellent correlation $(\mathrm{r}=0.98)$ and very good agreement with clinically acceptable bias of 5.4 degrees with 17.3 and -6.4 degrees for upper and lower 95\% confidence bound, respectively (BlandAltman Plot analysis, see figure 4).

Figure 4 illustrate the assessment of knee joint range of motion test in sitting position. Supine position may also be used to evaluate knee range of motion. Our pilot work on the comparison in measuring knee angles using video analysis and ARIADNE showed a bias of 5.4 degrees with 17.3 and -6.4 degrees for upper and lower bound, respectively (Bland-Altman Plot analysis, see figure 4), and significant 0.98 Pearson correlation coefficient.

\subsection{Age-related deterioration in postural balance using ARIADNE}

A pilot study investigate age-related changes in postural balance to determine the age at which balance deteriorates and falls risk increases. Twenty healthy adults (11 male, 16 female), divided into 5 age groups $(30-39,40-49,50-59,60-69)$ performed 30 seconds two-leg and one leg stance postural balance test using ARIADNE. Similar anteriorposterior and medi-lateral sway magnitude was found between the age groups when standing on two legs. During unilateral stance postural sway was greater in the older $(50-59,60-69)$ groups compared to the younger (30-39, 40-49) groups (see table 1). These results may suggest that postural sway may deteriorate from the age of 50 years thus may increase falls risk.

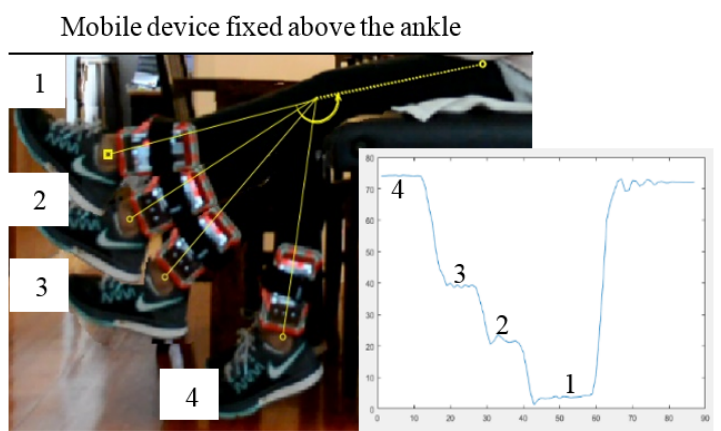

Figure 4. ARIADNE angles (bottom right) and video analysis angles while the patient extend their leg to 4 knee extension positions $(1,2,3,4)$.

Table 1: Mean \pm SD Medio-lateral and Anteriorposterior sway during 30 seconds double leg and single leg postural balance test (lower values indicates less sway).

\begin{tabular}{lcc}
\hline Age Group & $\begin{array}{c}\text { Medio- } \\
\text { lateral sway } \\
\text { (mean } \pm \text { SD) }\end{array}$ & $\begin{array}{c}\text { Anterior- } \\
\text { posterior sway } \\
\text { (mean } \pm \text { SD) }\end{array}$ \\
\hline $30-39$ & $0.008 \pm 0.002$ & $0.011 \pm 0.002$ \\
$40-49$ & $0.011 \pm 0.014$ & $0.009 \pm 0.010$ \\
$50-59$ & $0.009 \pm 0.010$ & $0.012 \pm 0.012$ \\
$60-69$ & $0.009 \pm 0.002$ & $0.015 \pm 0.005$ \\
\hline & Single Right Leg Balance \\
\hline $30-39$ & $0.017 \pm 0.013$ & $0.015 \pm 0.006$ \\
$40-49$ & $0.033 \pm 0.042$ & $0.024 \pm 0.028$ \\
$50-59$ & $0.062 \pm 0.096$ & $0.065 \pm 0.100$ \\
$60-69$ & $0.069 \pm 0.035$ & $0.046 \pm 0.012$ \\
\hline \multicolumn{3}{c}{ Single Left Leg Balance } \\
\hline $30-39$ & $0.029 \pm 0.016$ & $0.022 \pm 0.010$ \\
$40-49$ & $0.040 \pm 0.052$ & $0.027 \pm 0.033$ \\
$50-59$ & $0.057 \pm 0.069$ & $0.053 \pm 0.063$ \\
$60-69$ & $0.058 \pm 0.013$ & $0.046 \pm 0.012$ \\
\hline
\end{tabular}




\subsection{Methodology}

To test the proposed tele-assessment solution this section outlines the research plan. Teleassessment is the missing piece in telemedicine care for orthopaedic rehabilitation. Unique aspects of ARIADNE include that it: a) can provide remote, quantified, and postural and function control, and b) provide early detection of deviation, problems and potential complications. Our pilot study will serve to incorporate key co-design principles to ensure clinician and patient input in the design and development of ARIADNE, and then test the definitive solution in terms of: (a) desirability (patients and clinicians), and clinician and patient usability and acceptance, (b) reliability (ability to deliver consistently on key clinical outcomes) and "fit for purpose", and (c) cost-effectiveness.

The tele-assessment platform: ARIADNE is very simple to use as it integrates a web based database and interface platform and motion sensor data that is captured remotely from the patient's mobile phone while the patient performs their essential postural and functional measures, including [7]: 1) range of motion, 2) postural balance, 3) chair rise, 4) 40 meter fast paced walk, and 5) timed up and go (TUG). The motion sensor data is processed to objectively quantify patients' performance level.

Primary Hypotheses: (a) ARIADNE will be desirable for, and usable by, both patients and clinicians. (b) ARIADNE will be clinically reliable and will meet the key needs for both clinicians and patients, "fit for purpose".

Primary Aims: to (a) assess desirability and usability of ARIADNE. (b) assess clinical reliability including Minimum Detectable Change (MDC), and (c) evaluate if ARIADNE is fit for purpose.

Secondary Hypotheses: (a) ARIADNE will provide a cost-effective solution to support postsurgical recovery for THR and TKR patients. (b) ARIADNE will support the healthcare value proposition of better access, quality and value of care for THR and TKR contexts.

Secondary Aims: to (a) determine the costeffectiveness of ARIADNE. (b) demonstrate that ARIADNE supports a healthcare value proposition of better access, quality and value for THR and TKR patients.

The study outcomes will create ARIADNE, a new tele-assessment solution to address a current gap in telemedicine delivery, that is desirable and useable for patients and clinicians, clinically reliable, fit for purpose, and cost-effective with a high likelihood of addressing current issues around the sustainability of practice for THR and TKR patients. ARIADNE will be at the forefront in future telemedicine delivery models and will assist to advance Australia in telemedicine research, which unfortunately, to date, is lagging behind, having only $6.3 \%$ of the telemedicine usability research published in the world.

\subsection{Overview of Design}

To ensure a robust solution it is essential to conduct a feasibility pilot study to measure the desirability, usability, reliability, MDC, fit for purpose, and cost-effectiveness, and better access, quality and value of the tele-assessment platform for THR and TKR patients. The tele-assessment will be utilised during the conventional THR and TKR standard care pathway.

\subsection{Participants}

Patients: 76 patients ( 4 of whom will be solely involved in the solution co-design phase) from the Western Health outpatient clinic are to be recruited. On average 221 and 259 THR and TKR patients are treated each year at Western Health, respectively. Patients who are scheduled for THR and TKR as suggested by their consultant and meet the eligibility criteria will be invited to participate in the study by the researchers who will issue them with the information statement. Once the participant consents to participate, they will receive a written plan with booked dates for tele-assessment sessions with the responsible Community Based Rehabilitation (CBR) senior physiotherapists. Any participant considered to require surgery, such as revision, during the course of the study will be removed from the study. Inclusion criteria: (a) after THR or TKR, (b) have proficient English language skills (i.e. do not require an interpreter), (c) have a mobile smart phone. Exclusion criteria: (a) THR or TKR with clinical complications, (b) do not possess and use a mobile smart phone.

Clinicians: the Senior Fellow physiotherapist and 3 physiotherapists from CBR will be recruited to deliver the tele-assessment sessions. The clinicians will have substantial experience in standard care for joint replacement patients.

\subsection{Protocol}

The project will begin with the ethics submission and a final ARIADNE co-design session with 2 clinicians and 4 patients (2 TKR and 2 THR). Prior to tele-assessment at $\mathbf{T 0}$, clinicians and patients will participate in an educational focus group session. In this session participants will be educated on the use of ARIADNE with preparation for their joint replacement journey.

Tele-assessments will be performed on 10 occasions including; base-line pre surgery-1 (T1), pre surgery-2 (T2), and at 1 (T3), 2 (T4), 3 (T5), 4 (T6), 5 (T7), 6 (T8), 9 (T9), and 12 (T10) weeks post-surgery. The duration of each tele-assessment session is 20 minutes. In each session, the patient 
will start the app that automatically connects to the clinician web portal. The patient will then insert the mobile phone in the waist pouch and attach it around their waist. The clinician will instruct the patient to perform the tasks (balance, TUG, chair rise, range of motion) while the mobile app captures the motion data and automatically uploads it to the web portal for storage and further analysis. At $\mathbf{T} 2$ and $\mathbf{T 1 0}$ patient questionnaires will be administered to assess usability of ARIADNE. In addition, at T10 a clinician focus group will be conducted.

\subsection{Ethics and Sample Size}

Approval has been obtained from Swinburne University of Technology Ethics in Human Research Committee.

Sample size was calculated using the G*Power software tool [32] ${ }^{8}$ for repeated Analysis of Variance test $(2 \times 5$, age groups $\times$ tele-assessment sessions, allowing for $50 \%$ completion of 10 possible assessments) for both THR and TKR participants. This analysis is based on the teleassessment postural and functional outcome measures $(2 \mathrm{x} 10$, groups $\mathrm{x}$ tele-assessment sessions). The Cohen's effect size was based on a previously published RCT that investigated the effectiveness of tele-rehabilitation as a supplement to rehabilitation in THR and TKR patients [33] ${ }^{7}$. Patients that received standard care reported an improvement of 1.5 seconds in TUG having $9.0 \pm 2.4$ $\mathrm{s}$ and $7.5 \pm 1.6 \mathrm{~s}$ in pre and 3 weeks post-surgery testing, respectively. For the chair rise test an improvement of $3.9 \mathrm{~s}$ was found from pre and 3 weeks post-surgery, $17.1 \pm 6.2 \mathrm{~s}$ and $13.2 \pm 2.3 \mathrm{~s}$, respectively. Further, subjective parameters on function using the Western Ontario and McMaster Universities Arthritis Index were found to be 24.8 \pm 16.4 and $13.9 \pm 14.3$ for pre and 3 weeks postsurgery, respectively. The Cohen's d effect size using the above data were calculated to be 0.73 , 0.83 , and 0.71 . Using a 0.7 effect size $(f=.35)$, $\alpha=0.05$, and power $=0.80$, for repeated measures ANOVA 15 participants are needed in each age group, for both THR and TKR participants (60 total). Having $20 \%$ dropouts 72 participants in total will be recruited for this study.

\subsection{Results to Date}

The assessment of the fidelity, efficacy and fit for purpose of the developed ARIADNE solution requires many stages and is thus a longitudinal study. On the receipt of ethics approval, initial phases of the design science research methodology have been conducted with a small group of patients and clinicians respectively to fine tune the solution. This is an important key step to ensure high clinician and patient use as well as ensure the developed solution will support the required needs for rehab of THR and TKR patients. The ARIADNE solution now has patient and clinician approval and based on a small pilot study demonstrated ease of use and fit for purpose. While not statistically significant, this directional data provides support to progress to the next phase with confidence. The next key step is to conduct a large scale clinical trial to capture key data around the impact of the solution to support THR and TKR patients in their rehabilitation. Once the clinical trial is concluded it will then be possible to address issues around deployment of the solution into appropriate clinical contexts.

\subsection{Discussion}

ARIADNE is designed to remotely capture, analyse, and interpret body motion using the accelerometer and gyroscope motion sensors embedded in today's mobile phones. The mobile phones we have today have 3-axial accelerometer and gyroscope components. The accelerometer allows the measurement of linear acceleration in three orthogonal directions $(x, y$, and $z$ ) and the gyroscope allows the measurement of angular velocity in the $\mathrm{x}, \mathrm{y}$, and $\mathrm{z}$ axes. The linear acceleration and angular velocity signals can be processed and used to analyse body motion and further provide interpretation of the movement quantity and quality, such as level of stability during quiet standing. The ability to remotely connect to patient mobile phone and capture accelerometer and gyroscope data creates new opportunities for clinicians, sport trainers, and engineers to remotely quantify and analyse the performance level of any posture and/or movement task.

ARIADNE is comprised of 3 components including the mobile phone app, web based application, and a cloud database. The objective of the mobile phone app is to capture accelerometer and gyroscope data at $100 \mathrm{~Hz}$ and to upload the data to the cloud database. The objectives of the web based application is to provide the examiner (clinician, sport trainer) with a web browser platform to remotely connect and control the mobile phone app, instruct the mobile app to upload the accelerometer and gyroscope data to the cloud database, and to perform data analysis for further interpretation and reporting of the level of performance.

\subsection{Conclusions}

Our designed solution, ARIADNE, represents a novel and unique approach to telehealth rehabilitation in orthopaedic care for THR and TKR patients. To date, current telehealth solutions in this space do not address tele-assessment, which means that there is a significant limitation in the current 
post-operative critical 12-week period for THR and TKR patients. Hence, ARIADNE not only addresses this key void, but it serves to also potentially help to address a major conundrum facing healthcare delivery around THR and TKR; namely, the fact that current services will be unsustainable by 2030. Moreover, our solution is consistent with $2018 \mathrm{MoC}$ recommendation for "Innovation in service delivery model". By including a co-design approach and assessing ARIADNE as fit for purpose, we will have a unique tele-assessment solution that can be used for THR and TKR patients and potentially beyond, thereby also serving to leapfrog Australian telehealth initiatives. If the results of the clinical trial provide a positive endorsement for ARIADNE, then we would have successfully developed a unique teleassessment solution that addresses a key gap in post-surgical recovery for THR and TKR patients.

\section{Acknowledgment}

Funding for this work has been received by a grant from the Department of Defence and Epworth Medical Foundation.

\section{References}

[1] I.N. Ackerman, M.A. Bohensky, E. Zomer et al. 2019. The projected burden of primary total knee and hip replacement for osteoarthritis in Australia to the year 2030. BMC Musculoskelet Disord 20(1) 90.

[2] J.G. Nutt and M.C. Solan 2017. Ageing and orthopaedics. Orthopaedics and Trauma 31(5) 321-325.

[3] T.G. Russell, P. Buttrum, R.Wootton et al. 2011. Internet-based outpatient telerehabilitation for patients following total knee arthroplasty: a randomized controlled trial. J Bone Joint Surg Am 93(2) 113-120.

[4] The Brigham and Women's Hospital, I., Department of Rehabilitation Services 2010. Standard of Care: Total Hip Replacement.

[5] M.M. Jansson, M. Harjumaa, A.P. Puhto et al. 2020. Patients' satisfaction and experiences during elective primary fast-track total hip and knee arthroplasty journey: A qualitative study. J Clin Nurs 29(3-4) 567-582.

[6] E.K. Aasvang, I.E Luna, and H. Kehlet, H. 2015. Challenges in postdischarge function and recovery: the case of fast-track hip and knee arthroplasty. Br J Anaesth 115(6) 861-866.

[7] 2018, Victorian Model of Care for
Osteoarthritis of the Hip and Knee. Melbourne: MOVE muscle, bone \& joint health., by Victorian Musculoskeletal Clinical Leadership Group.

[8] Health, D.o. 2010, Elective Joint Replacement Service Model of Care., by Department of Health, W.A.

[9] T.G. Russell, P. Buttrum, R.Wootton, R. et . 2011. Internet-based outpatient telerehabilitation for patients following total knee arthroplasty: a randomized controlled trial. J Bone Joint Surg Am 93(2) 113-120.

[10] B. Klaassen, B.J. van Beijnum, and H.J. Hermens, 2016. Usability in telemedicine systems-A literature survey. Int J Med Inform 93 57-69.1

[11] B.R. Richardson, P.Truter, R. Blumke, R. et al. 2017. Physiotherapy assessment and diagnosis of musculoskeletal disorders of the knee via telerehabilitation. J Telemed Telecare 23(1) 88-95.

[12] S. Eichler, S. Rabe, a. Salzwedel et al. 2017. Effectiveness of an interactive telerehabilitation system with home-based exercise training in patients after total hip or knee replacement: study protocol for a multicenter, superiority, no-blinded randomized controlled trial. Trials 18(1) 438.

[13] N. Wickramasinghe and J. Schaffer 2018 Theories to Inform Superior Health Informatics Research and PracticeSpringer New York.

[14] T. Mettler 2016. Anticipating mismatches of HIT investments: Developing a viability-fit model for e-health services. International Journal of Medical Informatics, 85 Vol. 1, pp.104-115.

[15] A.K. Tjan, 2001. Finally, a way to put your internet portfolio in order, Harvard Business Review, Vol. 79 No. 2, pp. 76-85.

[16] T.P. Liang and C.P. Wei 2004. Introduction to the special issue: a framework for mobile commerce applications, International Journal of Electronic Commerce, Vol. 8 No. 3.

[17] D. Goodhue 1988 Development and Measurement Validity of a Task-Technology Fit Instrument for User Evaluations of Information System https:/www.researchgate.net/publication/2280 57362_Development_and_Measurement_Vali dity_of_a_Task- 
Technology_Fit_Instrument_for_User_Evaluat ions_of_Information_System

[18] C. Soh, S. Kien, J. and Tay-Yap, J. 2000 Enterprise Resource Planning: Cultural Fits and Misfits: Is ERP a Universal Solution? Communications of the ACM, 43 Vol. 4, pp. 47-51.

[19] S. Chatterjee, G. Moody, P.B. Lowry, et al. 2020. Information Technology and organizational innovation: Harmonious information technology affordance and courage-based actualization. The Journal of Strategic Information Systems, 29(1), p.101596.[20] E. Umble, R. Haft and M. Umble 2003. Enterprise Resource Planning: Implementation Procedures and Critical Success Factors, European Journal of Operational Research, 146, Vol. 2 pp. 241-257.

[21] T. G. Russell, P. Buttrum, R. Wootton, et al. 2011. Internet-based outpatient telerehabilitation for patients following total knee arthroplasty: a randomized controlled trial. J Bone Joint Surg Am 93(2) 113-120.

[22] Witchel, H.J., Oberndorfer, C., Needham, R. et al. 2018. Thigh-Derived Inertial Sensor Metrics to Assess the Sit-to-Stand and Stand-to-Sit Transitions in the Timed Up and Go (TUG) Task for Quantifying Mobility Impairment in Multiple Sclerosis. Front Neurol 9684

[23] N. Steinberg, R. Adams, G. Waddington, G. et al. 2017. Is There a Correlation Between Static and Dynamic Postural Balance Among Young Male and Female Dancers? J Mot Behav 49(2) 163-171.

[24] N. Steinberg, O. Tirosh, R. Adams, et al. 2017. Influence of Textured Insoles on Dynamic Postural Balance of Young Dancers. Med Probl Perform Art 32(2) 63-70.

[25] O. Tirosh, R. Baker and J. McGinley, J. 2010. GaitaBase: Web-based repository system for gait analysis. Comput Biol Med 40(2) 201-207.

[26] O. Tirosh, P. Tran, J. Renouf, et al. 2019. PROMsBase: Web-based repository portal for patient-reported outcome measures in orthopaedics. Health Informatics J 25(3) 867 877.

[27] O. Tirosh, G. Orland, A. Eliakim, et al. 2017. Tibial impact accelerations in gait of primary school children: The effect of age and speed. Gait Posture 57 265-269.
[28] O.Tirosh, G. Orland, A. Eliakim et al. 2019. Repeatability of tibial acceleration measurements made on children during walking and running. J Sci Med Sport 22(1) 91-95.

[29] O.Tirosh, G. Orland, A. Eliakim et al. 2020. Attenuation of Lower Body Acceleration in Overweight and Healthy-Weight Children During Running. J Appl Biomech 1-6.

[30] J.W.L.Keogh, A. Cox, S. Anderson et al. 2019. Reliability and validity of clinically accessible smartphone applications to measure joint range of motion: A systematic review. PLoS One 14(5) e0215806.

[31] M.H. Pham, E. Warmerdam, M. Elshehabi et al. 2018. Validation of a Lower Back "Wearable"Based Sit-to-Stand and Stand-to-Sit Algorithm for Patients With Parkinson's Disease and Older Adults in a Home-Like Environment. Front Neurol 9652.

[32] F. Faul, E. Erdfelder, A.G. Lang et al. 2007. $G^{*}$ Power 3: a flexible statistical power analysis program for the social, behavioral, and biomedical sciences. Behav Res Methods 39(2) 175-191.

[33] S. Eichler, A. Salzwedel, S. Rabe, S. et al. 2019. The Effectiveness of Telerehabilitation as a Supplement to Rehabilitation in Patients After Total Knee or Hip Replacement: Randomized Controlled Trial. JMIR Rehabil Assist Technol 6(2) e14236. 